\title{
Liquid nanoparticles: manipulating the nucleation and growth of nanoscale droplets
}

\author{
Ruoxu Wang, ${ }^{[a, b]}$ Fei Han, ${ }^{[a, b]}$ Bo Chen, ${ }^{[c]}$ Lingmei Liu, ${ }^{[d]}$ Shaoyan Wang, ${ }^{[a, b]}$ Hua Zhang, ${ }^{[e, f]}$ Yu Han, ${ }^{[d]}$ \\ and Hongyu Chen*[a]
}
[a] Prof. H. Chen, Dr. R. Wang, Dr. F. Han, Dr. S. Wang
Institute of Advanced Synthesis (IAS), and School of Chemistry and Molecular Engineering Nanjing Tech University
No.30 Puzhu Road(S), Nanjing, China
E-mail: iashychen@njtech.edu.cn
[b] Dr. R. Wang, Dr. F. Han, Dr. S. Wang
Division of Chemistry and Biological Chemistry, School of Physical and Mathematical Sciences
Nanyang Technological University
21 Nanyang Link, 637371, Singapore
[c] Dr. B. Chen
School of Materials Science and Engineering
Nanyang Technological University
50 Nanyang Avenue, 639798, Singapore
[d] Dr. L. Liu, Prof. Y. Han
Physical Science and Engineering Division
King Abdullah University of Science and Technology
Thuwal 23955-6900, Kingdom of Saudi Arabia
[e] Prof. H. Zhang
Department of Chemistry
City University of Hong Kong
83 Tat Chee Ave, Kowloon Tong, Hong Kong, China
[f] Prof. H. Zhang
Hong Kong Branch of National Precious Metals Material Engineering Research Center (NPMM)
City University of Hong Kong
83 Tat Chee Ave, Kowloon Tong, Hong Kong, China
Supporting information for this article is given via a link at the end of the document.

\begin{abstract}
By manipulating the nucleation and growth of solid materials, the synthesis of various sophisticated nanostructures has been achieved. Similar methodology, if applied to liquid, could enable the mass-production and control of ultra-small droplets at the scale of nanoparticles $\left(10^{-18} \mathrm{~L}\right.$ or below). It would be highly desirable since droplets play a fundamental role in numerous applications. Here we present a general strategy to synthesize and manipulate nanoscale droplets, similar to what has been done to solid nanoparticles in the classis solution-synthesis. It was achieved by a solute-induced phase separation which initiates the nucleation of droplets from a homogeneous solution. These liquid nanoparticles have great potentials to be manipulated like their solid counterparts, borrowing from the vast methodologies of nanoparticle synthesis, such as burst nucleation, seeded growth, and co-precipitation. Liquid nanoparticles also serve as a general synthetic platform, to fabricate nanoreactors, drug-loaded carriers, and other hollow nanostructures with a variety of shell materials.
\end{abstract}

\section{Introduction}

In the synthesis of solid nanoparticles (NPs), methods such as grinding and milling are deemed top-down, whereas solution growth is bottom-up. The latter offers control over particle shape, diameter, and size distribution, enabling the fabrication of sophisticated nanostructures. ${ }^{[1-4]}$ In contrast, there are few studies on bottom-up emergence of liquid droplets, particularly in controlling and manipulating the droplets in the process.
Liquid droplet underlies numerous fundamental processes in the realms of medicine, cosmetics, and catalysis. It has long been sought to push the droplet size to its minimum. The commonly used term nano- and pico-droplets are defined volume-wise $\left(10^{-}\right.$ $9-10^{-12} \mathrm{~L}$ ), with diameters around hundreds of micrometers. ${ }^{[5,6]}$ If droplets can be generated at a comparable size and uniformity as nanoparticles (NPs), i. e. liquid nanoparticles (LNPs), their volume could reach atto- to zepto-liters $\left(10^{-18}-10^{-21} \mathrm{~L}\right)$. Being such small size would open the potential for subcellular applications, let alone the tremendous surface area and the phase-transfer efficiency it brings. The synthesis of such LNPs calls for bottom-up methodologies. That is, transplanting the NP synthetic platform from solid to liquid.

In this work, a solute-induced phase separation (SIPS) process is developed in pursuit of this goal (Fig. 1B). Resembling the typical colloidal synthesis of solid NPs, ${ }^{[7-9]}$ SIPS initiates the nucleation and growth of liquid from a homogeneous liquid phase, leading to a bottom-up emergence of LNPs where intervention becomes possible. Trapping the LNPs serves as a general platform to one-step synthesize nanoreactors and nanocarriers loaded with various catalysts and drugs. Additionally, a library of hollow nanospheres made of oxides, polymers, and metal-organic framework (MOF) have been synthesized.

\section{Results and Discussion}


While it is well known that ethanol is infinitely miscible with water, a typical SIPS separates one from the other, more specifically, by adding an aqueous solution of $\mathrm{NaOH}$ into an ethanol solution of citric acid. The process is done under vortex mixing with hydroxypropyl cellulose (HPC) as a thickening agent. The resulting sodium citrate salt has a strong affinity with water yet incompatible with ethanol. It triggers a phase separation, from which the new aqueous phase emerges as salty droplets (Fig. $1 \mathrm{~B})$. The surface of these LNPs is passivated by silica shells from a modified Stöber reaction. ${ }^{[10]}$ As shown in the transmission electron microscopy (TEM) images, the resulting hollow silica nanospheres have diameters below $100 \mathrm{~nm}$ (Fig. 2E and S1A), implying the sub-attoliter volume of the liquid therein. Their narrow size distribution is visually confirmed and further supported by data of dynamic light scattering (DLS) and scanning electron microscopy (SEM, Fig. S1). Their size and uniformity rival that of the typical solid NPs and outcompete the hollow nanospheres from top-down methods. ${ }^{[11,12]}$

Since volume and mass are third power dependent on diameter, undesirable large particles, even at a small proportion, could take up significant amount of the total mass in the product. In the standard lognormal distribution, for example, the largest $10 \%$ particles contribute to over $95 \%$ of the total mass (Fig. S2). That is why uniformity is a major concern in the synthesis of nanoparticles and likewise for our LNPS.

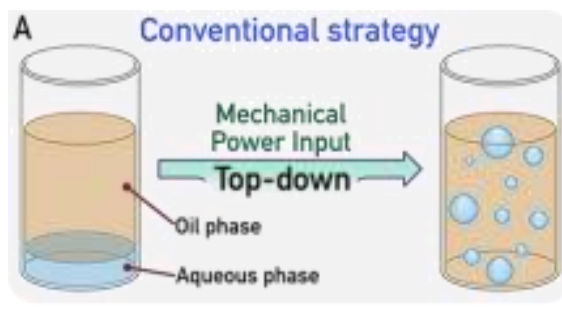

B

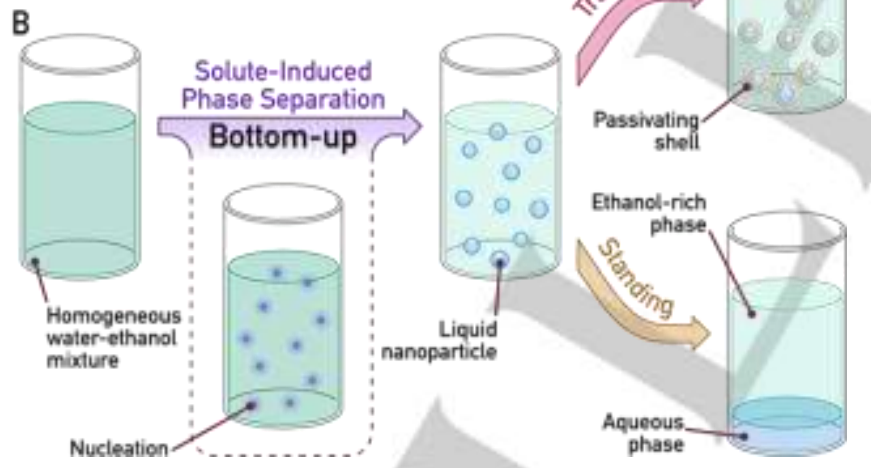

Figure 1. Comparison between the bottom-up and top-down method for generating droplets. (A) Schematics of a typical top-down method, where the input of mechanic power helps disperse an oil-water mixture into non-uniform droplets. (B) Schematics representing a bottom-up emergence of LNPs through a SIPS process, where a kosmotropic solute induces the phase separation from a water-ethanol mixture, initiating the nucleation and growth from the homogeneous liquid phase. Encapsulation of those LNPs leads to hollow nanospheres.

The nature of the emerging phase is investigated as follows. In the absence of HPC and the silica passivation, the SIPS led to a cloudy colloid. After prolonged standing, it gave two clear bulk liquid phases, with the ethanol-rich layer sitting on top. No solid precipitate was observed. Hence, the cloudiness indicates the existence of light-scattering liquid droplets. Furthermore, an HPC-free version of the typical SIPS process gave hollow intersecting spheres (Fig. 2F) indicative of a half-merged state of colliding droplets. These trapped intermediates further confirm the liquid nature of the initial NPs. It also proves that thickening agent is optional in the formation of LNPs. Its role is to deter collision, as opposed to the size-limiting effect of an amphiphilic surfactant. This substantiates the fundamental difference between LNPs and micelles, ${ }^{[13]}$ which size is thermodynamically controlled.

To shorten the shell-forming chemistry, tetraethyl orthosilicate (TEOS) was pre-mixed with ethanol before SIPS. Broken hollow spheres were observed in the product, apparently caused by the expansion of the LNPs therein. The hypothesis is consistent with their inflated diameter and the non-uniform shell thickness (Fig. S3). The remaining hollow spheres of smaller size also suggest that it is indeed possible to trap earlier intermediates.

When the passivation is postponed by $12 \mathrm{~h}$ after the initiation of LNPs (Fig. 2G), divergent products are obtained, with large hollow spheres (990 $\pm 220 \mathrm{~nm}$ ), and distinctively smaller ones $(120 \pm 45 \mathrm{~nm})$. In other words, over $99.9 \%$ of the liquid material exists in the form of large droplets, highlighting the great importance of size uniformity for LNPs. The silica shell is of obvious significance, as HPC could only provide limited suppression against merging. When polyvinylpyrrolidone (PVP) was introduced in the place of HPC, the SIPS process also gave hollow nanospheres $(180 \pm 40 \mathrm{~nm}$, Fig S4). They are significantly more uniform than the product from the thickeningfree experiments, and less so than the product of the typical process. It suggests that PVP is a valid thickening agent for the SIPS.

A common belief is that nanoscale droplets have too high surface energy in solution and thus, too transient to be trapped. Yet our success shows that the bottom-up approach could offer a window for trapping LNPs. The initial high concentration of poly(silicic acid) in a Stöber reaction may leads to fast nucleation on the interface, locking the surface of the LNPs, even though the further crosslink and hardening ${ }^{[10]}$ of the silica shell may take hours.

In the above experiments, the bulk aqueous phase evolved slowly from the "cloudiness": As the salt competes for water from the water/ethanol mixture, it leads to the enrichment of water molecules around the ions. [14,15] The resulting cluster (i.e. monomer) is then excluded from the homogeneous solution, triggering a minuscule phase separation. The individual aqueous LNPs would then grow in size (Fig. 2B, 2E), merge into larger droplets (Fig. 2F, 2G), and eventually converge to the macroscopic liquid phase. Hence, these steps of SIPS are similar to the nucleation, growth, and aggregation stages of the typical solid NP synthesis, ${ }^{[8]}$ where aggregation is often suppressed for uniform products. Solid NPs have a hard boundary, which can be further passivated by ligands and surfactants. ${ }^{[16,17]}$ Their collision is often reversible due to steric or charge repulsion (Fig. 2B). Being liquid, the LNPs lack such conveniences. Hence, the HPC is adopted to suppress their collision, and a hard shell is fabricated to passivate their surface. 


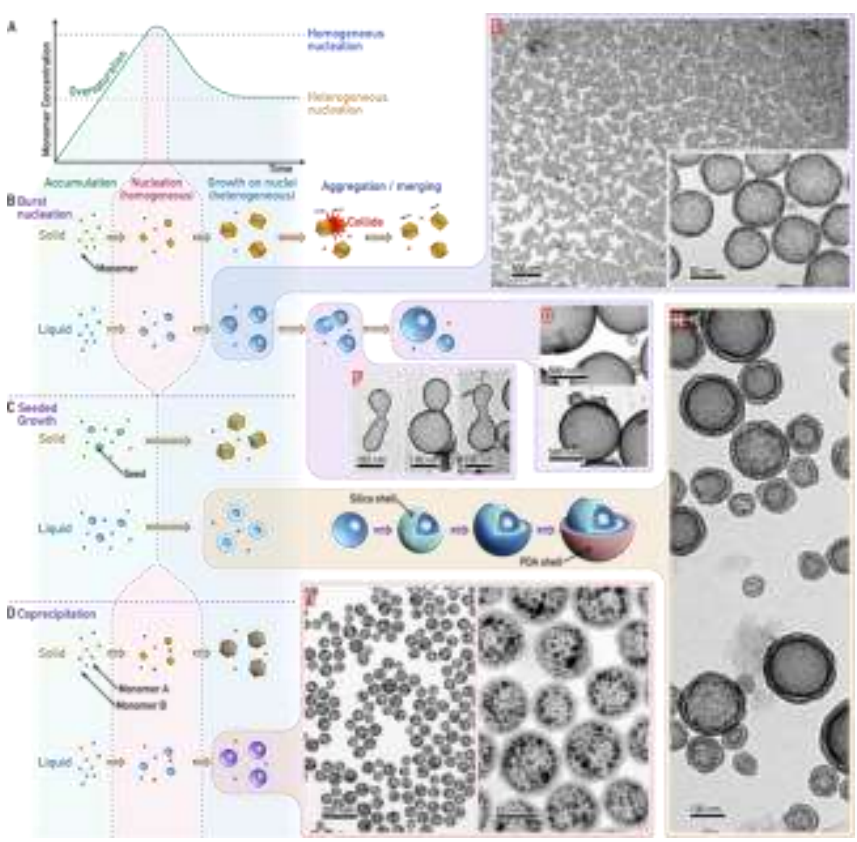

Figure 2. Manipulation of the nucleation and growth of LNPs like their solid counterparts. (A) Classical LaMer's model [7] depicting the nucleation and growth of nanoparticles: The concentration of monomer accumulates over time. When it becomes over-saturated, nuclei do not immediately appear until the critical concentration of homogeneous nucleation is reached. When the concentration drops below the critical concentration, only heterogeneous growth is allowed on the existing nuclei. (B) Comparison between the burst nucleation of solid and liquid NPs: When passivation is performed properly, uniform LNPs can be captured, as shown in E. In the later stage, the collision of solid nanoparticles is partially reversible, whereas LNPs could easily merge, as shown by $\mathbf{F}$ and $\mathbf{G}$, making passivation necessary. (C) Comparison of seeded growth between solid and liquid NPs: With pre-introduced seeds, homogeneous nucleation is inhibited, and monomers would selectively deposit on the seeds. In contrast to solid NPs, the boundary of LNPs after seeded growth is not observable, hence multi-step passivation is necessary, as shown by $\mathbf{H}$. (D) Comparison of the co-precipitation and the co-SIPS process: When a SIPS is induced by a strong kosmotrope, the formed nuclei would enrich the monomer of the other solutes, carrying them into the emerging LNPs. TEM images of $(\mathbf{E})$ the resulting silica hollow nanospheres after drying the encapsulated LNPs; $(\mathbf{F})$ captured intermediates of merging LNPs; $(\mathbf{G})$ the products after extensive merging; $(\mathbf{H})$ inorganic-organic hybrid nanomatryoshkas from the seeded growth of LNPs, with silica and PDA shells by consecutive passivation; (I) $\mathrm{Au}$ (III)-loaded nanocarriers synthesized from a coSIPS process.

Given the similarity between the liquid and solid NP synthesis, inspirations can be drawn from the latter. More specifically, a mild oversaturation of the growth material is always preferred (Fig. 2A), so that a short nucleation stage would set a narrow starting point (the "burst nucleation") for all growing particles. ${ }^{[8,18]}$ Hence, the NPs reach similar sizes after a same growth period. SIPS can also be seen as the oversaturation of liquid material from the initial liquid phase, and thus a mild SIPS would be preferred. The above standard procedure has already been optimized, where the SIPS is attained by the use of precursors - acid and base - instead of salt itself. It results in a smooth transformation to cloudiness in several seconds, suggesting a relatively mild SIPS. In contrast, when sodium citrate solution is directly added to ethanol, the mixture turns cloudy instantly (Fig. S5). TEM images shows that the product is less uniform than that of the standard procedure, which is further supported by the DLS data (Fig. S6).

A well-established strategy in solid NP synthesis is the "seeded growth", where deposition on the existing seeds would be preferred over the homogeneous nucleation in the solution. It enables selective and multi-step modification of the seeds to create sophisticated nanostructures. ${ }^{[19-21]}$ To emulate the process (Fig. 2C), the silica-passivated LNPs were used as seeds to accumulate more water. With the expansion of the LNP, its boundary was pushed beyond the permeable and robust silica shell, and polydopamine (PDA, vide infra) was formed to passivate the new surface. ${ }^{[22]}$ Figure $2 \mathrm{H}$ shows the resulting hybrid nano-matryoshkas with concentric shells and discernible gap in between, which serves as a clear evidence for the seeded growth. It suggests that homogeneous nucleation was inhibited, so that the additional water attached selectively to the existing "seeds" via heterogeneous nucleation.

Hybrid nanoshells are of great interest for the complementary properties provided by the inorganic-organic composite. ${ }^{[23,24]}$ On the other hand, gapped multishells are known for their ultra-high surface area in gas harvesting and battery applications. ${ }^{[25-27]}$ From these perspectives, our facile and direct route to the hybrid nano-matryoshka is of significance.

The elemental step of the SIPS is the exclusion of the watersurrounded ion clusters (monomers), where the free energy change can be described as:

$\Delta G=-\Delta G_{\text {org }}+\Delta G_{\text {water }}-\frac{n}{N_{A}} \Delta G_{\text {mix }}$ (1),

where $\Delta G_{\text {org }}$ and $\Delta G_{\text {water }}$ are the free energy of solvation for the ion in the organic solvent and in water (hydration), and $\Delta G_{\text {mix }}$ is the free energy of mixing water with the organic solvent. $N_{A}$ is the Avogadro constant and $n$ is the number of water molecules in the ion cluster. In a given experiment, the $\Delta G_{\text {mix }}$ term is a constant. The solvation energy can be determined by the Born's approximation: ${ }^{[28,29]}$

$\Delta G_{\text {solvation }}=-\left(1-\frac{1}{\varepsilon_{\text {out }}}\right) \frac{q^{2}}{2 R}(2)$,

where the ion is approximated as a hard sphere with charge $q$ and radius $R$. It is embedded into a dielectric continuum with the constant of $\varepsilon_{\text {out. }}$ Thus, the spontaneous process of SIPS gives:

$\left(\frac{1}{\varepsilon_{\text {water }}}-\frac{1}{\varepsilon_{\text {org }}}\right) \frac{q^{2}}{2 R}<\frac{n}{N_{A}} \Delta G_{\text {mix }}$ (3).

In other words, the ion is more favorable in water than in the organic solvent. The difference between the solvation energies is competing for water from the mixed solution. For a typical solvent, $\varepsilon_{o r g}$ is smaller than $\varepsilon_{w a t e r}$. Therefore, the main variables are $q^{2} / 2 R$, meaning that the SIPS would favor ions with higher charge and lower radius.

Such a trend resembles the ion ranking in the Hoffmeister series, ${ }^{[30]}$ which is initially adopted to rate the ability of ions in precipitating proteins (Fig. 3A). Its concept has since been expanded to incorporate more situations. ${ }^{[31]}$

Following this interpretation, a series of water-soluble salts have been tested in the place of sodium citrate. Anions ranked higher in the Hoffmeister series, such as sulfate, phosphate, and oxalate were all able to produce LNPs, which after passivation were characterized as hollow silica nanospheres (Fig. 3B, 3C, 3D). On the other hand, lower ranked anions such as iodide, 
acetate, and nitrate have low charge density. They are relatively more soluble in ethanol suggesting significant $\Delta G_{\text {org. }}$. As expected, they are not suitable for inducing SIPS.

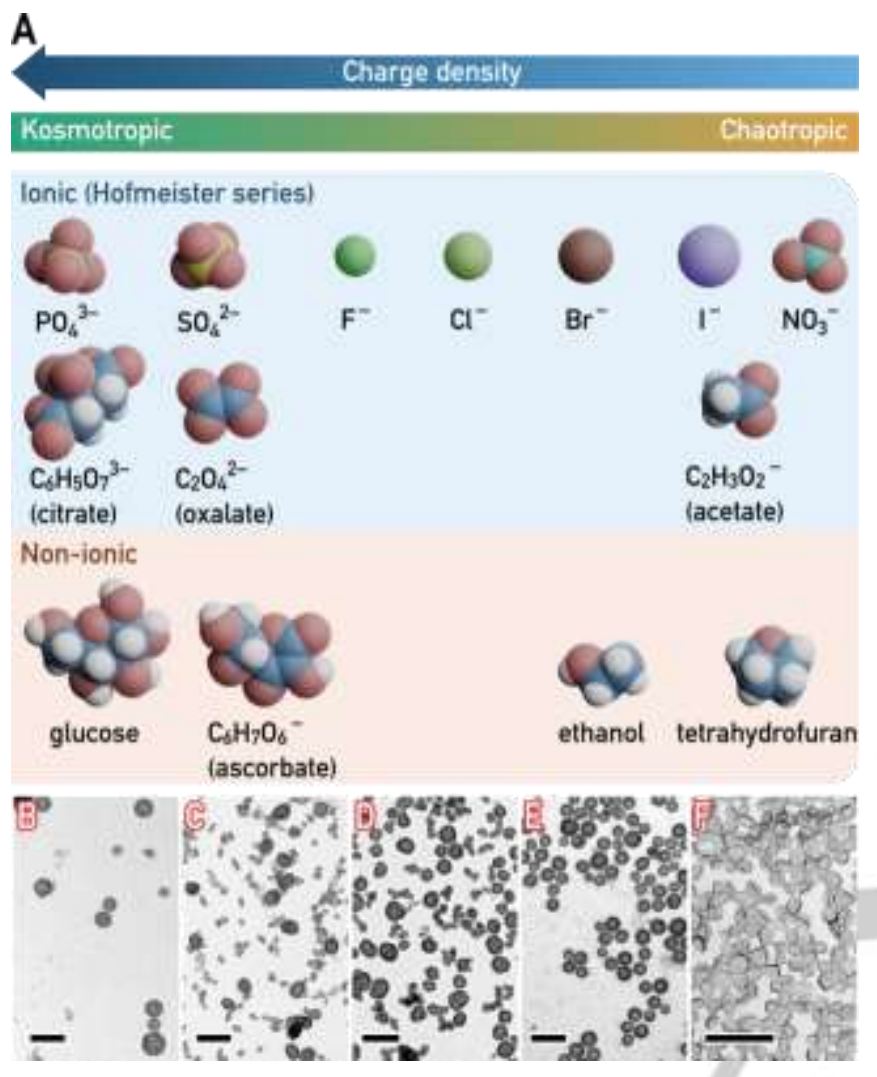

Figure 3. Concepts of the generalized kosmotrope and chaotrope in SIPS. (A) Strength of kosmotropicity follows the Hofmeister series which prefers anions with higher charge density. Carbohydrate such as glucose and ascorbate are also kosmotropes, whereas organic solvents such as ethanol and tetrahydrofuran are chaotropes. TEM images of the products from the SIPS processes triggered by different kosmotropes: (B) phosphate; (C) sulfate; (D) oxalate; (E) glucose; and (F) ascorbate. Scale bars are $200 \mathrm{~nm}$.

Related to the Hoffmeister series, the concept of kosmotrope and chaotrope is more broadly applied in studying proteins and water structures. ${ }^{[32-34]}$ Kosmotrope literally means "order maker", which promotes the hydrogen bonding or intermolecular networks in an aqueous mixture. The vast majority of soluble salts and carbohydrates are kosmotropic to a certain extent. The opposite of kosmotrope, namely "chaotrope" (Fig. 3A), weakens those interactions, including most of the water-miscible organic solvents. ${ }^{[33]}$ Based on their correlated theory, extensive techniques have been developed to separate and purify biomolecules. ${ }^{[35,36]}$ From this perspective, the SIPS can be described as such a process, where the introduction of a kosmotrope induces the phase separation in a mixture of water and a chaotrope. It is reasonable to expect a high universality for SIPS in similar ternary systems.

Non-ionic chemicals such as carbohydrates are also deemed kosmotropes. In a modified reaction, a glucose solution was introduced to trigger the SIPS in the place of salts, marked by the gradual cloudy transformation of the mixture. After the silica passivation, it gave hollow nanospheres verified by TEM (Fig $3 \mathrm{E}$ ). The above procedure is also compatible with sucrose (Fig. S7). Sodium ascorbate, as a carbohydrate-derived salt, could also generate LNPs (Fig. 3F). Considering its low charge density, the strong kosmotropicity likely comes from its carbohydrate identity.

Cations generally have a minor effect in SIPS, even for those with multiple charges. It agrees with the observations in the kosmotrope-chaotrope literature, likely because of their stronger affinity with ethanol. For chaotropes, water-miscible organic solvents such as tetrahydrofuran, acetonitrile, and dimethylformamide have been tested in the place of ethanol and found compatible (Fig. S8).

With the SIPS process, the product is by definition an encapsulated, kosmotrope-loaded droplet. It could serve as a powerful synthetic platform, as demonstrated by our initial attempts of loading catalysts and drugs. Thus, the LNPs become nanoreactors for catalytic heterogenization, and nanocarriers for drug delivery. Traditionally, fabrication of such functional hollow nano-objects requires complicated procedures. Take the nanocarrier as an example:[37,38] Firstly, a nanoparticle is synthesized as a sacrificial core, on which a layer of shell is deposited. The core is then removed by etching, and the drug is loaded into the cavity by a lengthy diffusion process. Notably, permeability of the shell material is a necessity since it governs both etching and loading. It also brings along drug leaking since diffusion happens both ways, further complicating the drug release control. The SIPS process provides an alternative path to circumvent such hassles and restrictions. Without the need of etching, the choice of shell materials is broadened, and the whole procedure compressed into a one-step reaction. Especially, the drug release can be decoupled from the subtle balance between loading and leaking, facilitating functional design.

The loaded catalyst or drug is not limited to mere strong kosmotropes. A weak one can be carried together into the LNPs by a strong kosmotrope (the co-SIPS method), similar to the coprecipitation strategy (Fig. 2D). Trapping such LNPs could serves as a general loading platform for a large variety of watersoluble chemicals.

Reactive precursors of $\mathrm{Au}(\mathrm{III}), \mathrm{Ag}(\mathrm{I}), \mathrm{Cu}(\mathrm{II}), \mathrm{Pt}(\mathrm{IV}), \mathrm{Pd}(\mathrm{II})$, and $\mathrm{Co}(\mathrm{II})$, have been loaded into the nanoreactors. All of the isolated products, when re-dispersed in ethanol, showed colors of their corresponding complexes, suggesting that the metals were loaded in their ionic forms (Fig. 4C). When centrifuged again, all colors were concentrated at the bottom, leaving colorless supernatants. The precipitates can be repeatedly redispersed even after 5 cycles of centrifugation-dispersing process, showing that the liquid domains are stable and that the metals ions have been successfully heterogenized. After drying, their TEM images showed uniform hollow nanospheres with dark spots inside. Take the Au(III)-loaded nanoreactors as an example (Fig. 2l), the uniform size is further supported by DLS and SEM images (Fig. S9A). Its energy dispersive X-ray (EDX) mapping of elemental $\mathrm{Au}$ fits the bright spots in the corresponding High-Angle Annular Dark-Field Scanning 
Transmission Electron Microscopy image (HAADF STEM, Fig. 4A). To avoid the contamination caused by electron beam exposure, the nanoreactors were sintered at $650^{\circ} \mathrm{C}$. The sample turned from yellow to red, indicating the reduction of $\mathrm{Au}$ (III) salts to $A u(0)$ nanoparticles, which is further supported by the significant change of their powder X-Ray Diffraction (XRD) patterns (Fig. S9B). EDX mapping showed that the signals of $\mathrm{Si}$ and $\mathrm{O}$ elements coincide with the shell, where $\mathrm{Au}$ appeared as granules attached to the inner wall (Fig. 4B). Additionally, $\mathrm{NaBH}_{4}$ reduction has been applied to the $\mathrm{Au}(\mathrm{III})$-loaded nanoreactors, turning the dispersion from yellow to red. TEM images showed yolk-shell nanospheres with Au NPs inside the silica shells (Fig. $\mathrm{S} 9 \mathrm{C}$ ). All these experiments suggest that reactive Au precursors have been successfully loaded into the nanoreactors.

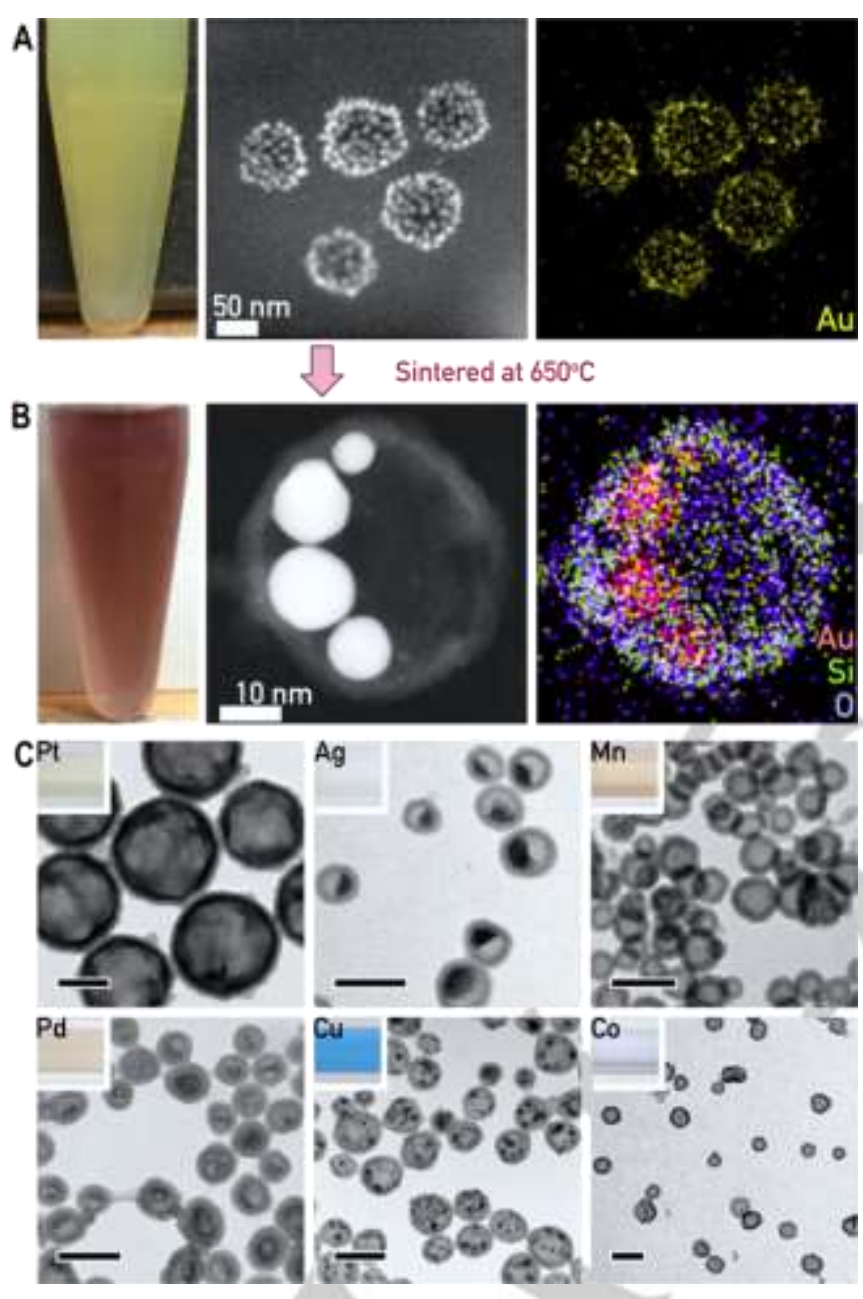

Figure 4. Nanoreactors synthesized from the SIPS processes. Scale bars are $100 \mathrm{~nm}$ unless specified. (A) Au(III)-loaded nanoreactors before sintering and its EDX mapping. (B) Au(III)-loaded nanoreactors after sintering and its EDX mapping. (C) Color and TEM images of the nanoreactors loaded with $\mathrm{Pt}(\mathrm{IV})$, $\mathrm{Ag}(\mathrm{I}), \mathrm{Mn}(\mathrm{II}), \mathrm{Pd}(\mathrm{II}), \mathrm{Cu}(\mathrm{II})$, and $\mathrm{Co}(\mathrm{II})$.

Anticancer drugs such as doxorubicin and cisplatin have also been loaded into nanocarriers through the SIPS process. In the case of doxorubicin, the product bears the characteristic bright orange color of the drug, which is further verified by UV-Vis data (Fig. S10). After centrifugation, no orange tint was observed in the supernatant, leaving a dark orange precipitate which can be readily re-dispersed. Cisplatin was similarly loaded. To verify its existence, the isolated product was washed, and then removed of the silica shell. Its absorbance around $210 \mathrm{~nm}$ marks the existence of the loaded cisplatin (Fig. S11).

Aside from silica, a large variety of materials can be made as shells. Once proper reactions are adopted to trap the LNPs, polymers, oxides, insoluble salts, and MOF material are all viable candidates. Polydopamine (PDA) is a versatile biocompatible material. In the place of TEOS, dopamine was introduced as its oleate for solubility in ethanol. To promote the polymerization of dopamine without interfering with the SIPS, ${ }^{[39]}$ $\mathrm{Nal}$ was added along with $\mathrm{NaOH}$ for its weak kosmotropicity. The resulting colloid gradually turned from amber to the dark brown color of PDA. TEM images of the product showed shrunken nanospheres with uniform size, likely due to the flexibility of the PDA shell (Fig. $5 \mathrm{C}$ ). In addition, the thermoset polymer phenol-formaldehyde resin is also technically compatible (Fig. S12). Its slow polymerization and the need for heat curing led to hollow spheres of a larger diameter and less uniformity. Their shape is similar to Figure $2 \mathrm{G}$, consistent with the extensive merging of the LNPs after a prolonged shellforming reaction.

Biocompatible inorganic materials such as calcium phosphate can be made into shells. The SIPS was induced by adding aqueous $\mathrm{NH}_{3}$ solution into an ethanol solution of phosphoric acid, indicated by the cloudiness after mixing. Then, an ethanolsoluble calcium precursor $\left(\mathrm{CaCl}_{2}\right)$ was introduced, so that the two reactants could approach the ethanol-water interface from both sides. The resulting hollow nanospheres were verified by TEM (Fig. 5D).

In addition to all of the above alkaline-based reactions, the SIPS is also compatible with acidic conditions, which is crucial if basesensitive ingredients are involved. For example, making magnetic shells would require Fe precursors, which would precipitate instantly in an alkaline SIPS. An acidic SIPS was triggered by adding aqueous sulfuric acid into an ethanol solution with sodium cations ( $\mathrm{NaOAc})$. $\mathrm{Fe}^{3+}$ were preloaded in the LNPs via the co-SIPS. The shell was formed by the addition of concentrated $\mathrm{NaOH}$ solution, converting the $\mathrm{Fe}^{3+}$ into oxides at the water-ethanol interface. Brown product formed instantly, which can be attracted by external magnet. The TEM image showed hollow nanospheres with an excellent uniformity (52 \pm $8 \mathrm{~nm}$, Fig. 5E, S13A), among the best in our samples. During the reaction, a gentle mixing was crucial to prevent the tearing of the initial weak shells (Fig. S13B). Given the fast reaction, it supports the aforementioned assumption that a fast shellforming reaction would benefit the uniformity. The powder XRD data of the product suggested that it is amorphous (Fig. S13C). $X$-Ray photoelectron spectrum (XPS) identified the iron in the product to be $\mathrm{Fe}(\mathrm{III})$ only (Fig. S13D). Elemental analysis from the inductively coupled plasma mass spectroscopy (ICP-MS) showed an iron content of $68.8 \%$ of the total weight, suggesting it to be $\mathrm{Fe}_{2} \mathrm{O}_{3}$.

To create MOF shells, we adopted the HKUST-1, ${ }^{[40]}$ a complex of copper and benzenetricarboxylic acid $\left(\mathrm{H}_{3} \mathrm{BTC}\right)$, as the model system. The SIPS was triggered by the kosmotropicity of $\mathrm{Cu}$ (II) sulfate. The $\mathrm{H}_{3} \mathrm{BTC}$ ligand was introduced from the ethanol 
phase. As $\mathrm{Cu}(\mathrm{II})$ and the ligand met at the water-ethanol interface, a layer of HKUST-1 formed, passivating the LNPs [41]. The products appear as hollow nanospheres under TEM (Fig. $5 \mathrm{~F})$, of which the powder XRD data confirms the assignment of HKUST-1 (Fig. S14).

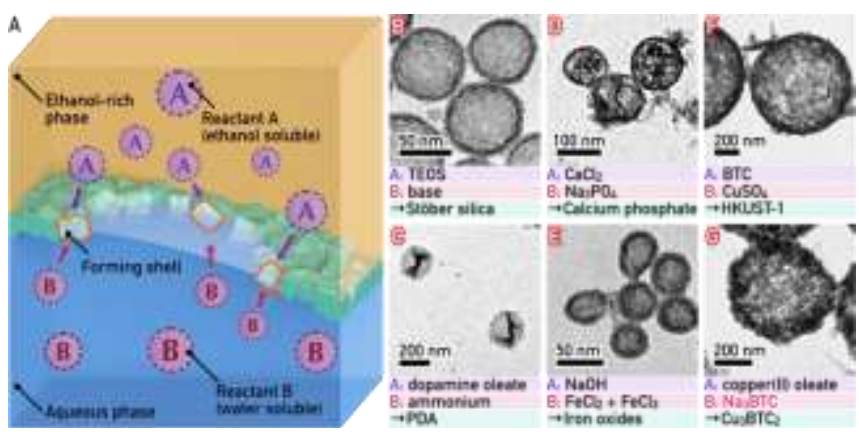

Figure 5. Schematics of a generalized shell-forming reaction and the various shells of different materials. (A) Schematics describing the design of a shellforming process: Reactants $a$ and $b$ are dissolved in the ethanol-rich phase and the aqueous phase, respectively. They react on the water-ethanol interface, forming a shell to passivate the LNP. Hollow nanospheres and their specific reactants $a$ and b for: (B) Stöber silica; (C) PDA; (D) calcium phosphate; (E) $\mathrm{Fe}_{2} \mathrm{O}_{3} ;(\mathbf{F})$ HKUST-1; and (G) $\mathrm{Cu}_{3} \mathrm{BTC}_{2}$.

The design of the shell-forming chemistry is summarized in Fig. $5 \mathrm{~A}$. The reactants should approach the target interface from both sides in the dissolved form, so that the smooth concentration gradient would lead to a controllable deposition. In contrast, insoluble reactants such as droplets or particles would only deliver "quantized" portions and thus unreliable for the shell-forming process. The general principles would allow versatile designs. For example, BTC could also be enriched inside the LNP as a trisodium salt, given its relatively high charge density and kosmotropicity. The shell was then formed by introducing $\mathrm{Cu}(\mathrm{II})$ oleate from the ethanol phase. The resulting product has thicker and coarser walls (Fig. 5G), distinctively different from the product of the previous reaction. Powder XRD data showed a smooth curve (Fig. S15), suggesting an amorphous complex. Its thermogravimetric analysis (TGA, Fig. S16) data shows that after $200{ }^{\circ} \mathrm{C}$ its normalized weight loss fits that of Basolite $\mathrm{C} 300,,^{[41]}$ a commercialized product of the HKUST-1. Since the initial weight loss of HKUST-1 comes from its embedded water, ${ }^{[40]}$ the shell material could be the solvent-free form of $\mathrm{Cu}_{3} \mathrm{BTC}_{2}$.

\section{Conclusion}

In summary, the LNPs are more than mere ultra-small droplets. With similar nucleation and growth as those of solid NPs, the emerging LNPs could be readily manipulated, as demonstrated in the burst nucleation and seeded growth. With the kosmotropechaotrope theory, the co-SIPS technique, and the various shellforming chemistry, the new strategy offers a broad generality for synthetic designs. While our initial steps of emulation have shown to be fruitful, considering the knowledge base of solid NP synthesis, there ought to be plenty of room to expand the synthetic freedom of LNPs.

\section{Acknowledgements}

The authors thank the Facility for Analysis Characterisation Testing and Simulation of the Nanyang Technological University for access to the facilities. Funding: This work is support by the National Natural Science Foundation of China (No. 21673117), Recruitment Program of Global Experts, Jiangsu Provincial Foundation for Specially Appointed Professor, Start-up fund of Nanjing Tech University (39837102), and SICAM Fellowship from Jiangsu National Synergetic Innovation Center for Advanced Materials. Ministry of Education Academic Research Fund (AcRF) Tier 1: RG10/16, RG111/15, Singapore. H.Z. thanks the financial support from ITC via Hong Kong Branch of National Precious Metals Material Engineering Research Center (NPMM), and the start-up grant (Project No. 9380100) and grants (Project No. 9610478 and 1886921) in City University of Hong Kong. Author contributions: R.W., F. H., and H.C. conceived the concept. R.W. designed the experiments and wrote the manuscript. L. L., B. C., H. Z., and Y. H. carried out the EDX measurement. S.W. helped data collection of the TGA and powder XRD. H. C. supervised the research and revised the manuscript. Competing interests: Authors declare no competing interests. Data and materials availability: All data is available in the main text or the supplementary materials.

Keywords: Nanoparticle • Nucleation • Hofmeister series • Kosmotropicity $\cdot$ Nanoreactor

[1] J. Park, K. An, Y. Hwang, J.-G. Park, H.-J. Noh, J.-Y. Kim, J.-H. Park N.-M. Hwang, T. Hyeon, Nature Materials 2004, 3, 891-895.

[2] X. Wang, J. Zhuang, Q. Peng, Y. Li, Nature 2005, 437, 121-124.

[3] L. Zhang, L. T. Roling, X. Wang, M. Vara, M. Chi, J. Liu, S.-I. Choi, J.

Park, J. A. Herron, Z. Xie, M. Mavrikakis, Y. Xia, Science 2015, 349, 412-416.

[4] W. H. Chong, L. K. Chin, R. L. S. Tan, H. Wang, A. Q. Liu, H. Chen,

Angewandte Chemie International Edition 2013, 52, 8570-8573.

[5] T. B. Jones, M. Gunji, M. Washizu, M. J. Feldman, Journal of Applied Physics 2001, 89, 1441-1448.

[6] P. Y. Colin, B. Kintses, F. Gielen, C. M. Miton, G. Fischer, M. F. Mohamed, M. Hyvönen, D. P. Morgavi, D. B. Janssen, F. Hollfelder, Nature Communications 2015, 6, 10008 .

[7] V. K. Lamer, R. H. Dinegar, Journal of the American Chemical Society 1950, 72, 4847-4854.

[8] Y. Wang, J. He, C. Liu, W. H. Chong, H. Chen, Angewandte Chemie International Edition 2014, 54, 2022-2051.

[9] S. Wu, Y. Sun, Nano Res 2019, 12, 1339-1345.

[10] X. Song, T. Ding, L. Yao, M. Lin, R. L. Siew Tan, C. Liu, K. Sokol, L. Yu, X. W. D. Lou, H. Chen, Small 2015, 11, 4351-4365.

[11] B. T. T. Pham, D. Nguyen, V. T. Huynh, E. H. Pan, B. Shirodkar-

Robinson, M. Carey, A. K. Serelis, G. G. Warr, T. Davey, C. H. Such, B. S Hawkett, Langmuir 2018, 34, 4255-4263.

[12] W. Wichaita, D. Polpanich, T. Suteewong, P. Tangboriboonrat, Polymer 2016, 99, 324-331.

[13] H. Cabral, K. Miyata, K. Osada, K. Kataoka, Chem Rev 2018, 118, 6844-6892.

[14] X. B. Wang, X. Yang, J. B. Nicholas, L. S. Wang, Science 2001, 294, $1322-1325$

[15] D. Paschek, R. Ludwig, Angewandte Chemie - International Edition 2011, 50, 352-353.

[16] M. R. Langille, M. L. Personick, J. Zhang, C. A. Mirkin, Journal of the American Chemical Society 2012, 134, 14542-14554.

[17] R. D. Tilton, $Æ$ E. G. v Lowry, 2008, 10, 795-814

[18] C. B. Whitehead, S. Özkar, R. G. Finke, Chemistry of Materials 2019, 31, 7116-7132.

[19] Y. Feng, Y. Wang, J. He, X. Song, Y. Y. Tay, H. H. Hng, X. Y. Ling, H. Chen, Journal of the American Chemical Society 2015, 137, 7624-7627.

[20] Z. Wang, B. He, G. Xu, G. Wang, J. Wang, Y. Feng, D. Su, B. Chen H. Li, Z. Wu, H. Zhang, L. Shao, H. Chen, Nature Communications 2018, 9, 563.

[21] L. Zhu, H. Wang, X. Shen, L. Chen, Y. Wang, H. Chen, Small 2012, 8 1857-1862.

[22] H. Lee, S. M. Dellatore, W. M. Miller, P. B. Messersmith, Science 2007, 318, 426-430.

[23] F. Caruso, R. A. Caruso, H. Möhwald, Science 1998, 282, 1111-1114. 
[24] J. Shi, Y. Jiang, X. Wang, H. Wu, D. Yang, F. Pan, Y. Su, Z. Jiang,

Chemical Society Reviews 2014, 43, 5192-5210.

[25] J. Wang, J. Wan, N. Yang, Q. Li, D. Wang, Nature Reviews Chemistry 2020, 4, 159-168.

[26] J. Qi, X. Lai, J. Wang, H. Tang, H. Ren, Y. Yang, Q. Jin, L. Zhang, R. Yu, G. Ma, Z. Su, H. Zhao, D. Wang, Chemical Society Reviews 2015, 44, 6749-6773.

[27] Y. J. Wong, L. Zhu, W. S. Teo, Y. W. Tan, Y. Yang, C. Wang, H. Chen, Journal of the American Chemical Society 2011, 133, 11422-11425.

[28] A. v. Onufriev, D. A. Case, Annual Review of Biophysics 2019, 48,

275-296.

[29] M. Born, Zeitschrift für Physik 1920, 1, 45-48.

[30] M. W. W. Kim D Collins, Quarterly Review of Biophysics 1985, 18, 323-422.

[31] K. I. Assaf, W. M. Nau, Angewandte Chemie - International Edition 2018, 57, 13968-13981.

[32] M. Chaplin, n.d.

[33] P. Ball, J. E. Hallsworth, Phys. Chem. Chem. Phys. 2015, 17, 82978305.

[34] J. P. Chin, J. Megaw, C. L. Magill, K. Nowotarski, J. P. Williams, P.

Bhaganna, M. Linton, M. F. Patterson, G. J. C. Underwood, A. Y. Mswaka, J.

E. Hallsworth, Proceedings of the National Academy of Sciences of the United States of America 2010, 107, 7835-7840.

[35] I. Katoh, S. Sato, N. Fukunishi, H. Yoshida, T. Imai, S. I. Kurata, Cell Research 2008, 18, 1210-1219.

[36] I. Bischofberger, D. C. E. Calzolari, P. de Los Rios, I. Jelezarov, V.

Trappe, Scientific Reports 2014, 4, 1-7.

[37] S. M. Kim, M. Jeon, K. W. Kim, J. Park, I. S. Lee, Journal of the

American Chemical Society 2013, 135, 15714-15717.

[38] X. Fang, C. Chen, Z. Liu, P. Liu, N. Zheng, Nanoscale 2011, 3, 16321639.

[39] S. Hong, Y. Wang, S. Y. Park, H. Lee, Science Advances 2018, 4 eaat7457.

[40] S. S. Y. Chui, S. M. F. Lo, J. P. H. Charmant, A. G. Orpen, I. D.

Williams, Science 1999, 283, 1148-1150.

[41] R. Ameloot, F. Vermoortele, W. Vanhove, M. B. J. Roeffaers, B. F.

Sels, D. E. de Vos, Nature Chemistry 2011, 3, 382-387.

[42] G. Majano, J. Pérez-Ramírez, Advanced Materials 2013, 25, 1052- 


\section{Entry for the Table of Contents}

Insert graphic for Table of Contents here.
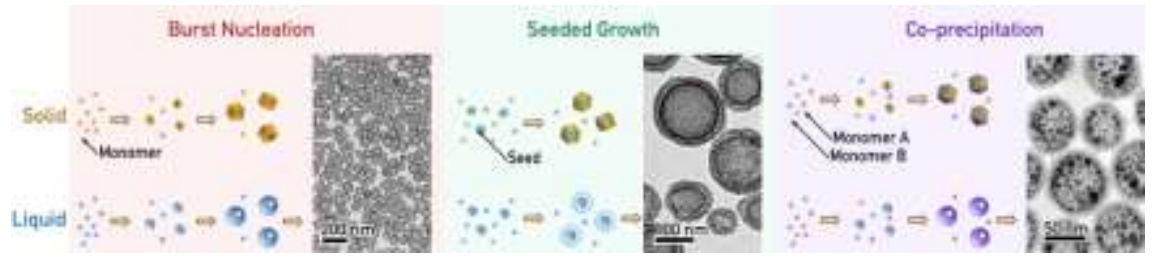

Manipulation of liquid droplet like nanoparticles: To mimic the classic synthetic methods of nanoparticles, a bottom-up methodology of fabricating nanoscale droplets has been developed. Synthetic techniques of solid nanoparticles, such as burst nucleation, seeded growth and co-precipitation, have been transplanted to liquid droplets. It leads to facile fabrication of multishell hollow structures, nanoreactors and carriers. 